\title{
Comparison of Barefoot vs. Shod Gait on Spinal Dynamics Using DIERS Formetric 4D and DIERS Pedoscan Systems
}

\author{
Carolyn Draus $^{1 *}$, David Moravec ${ }^{1 *}$, Adam Kopiec ${ }^{1}$, Patrick Knott ${ }^{2}$ \\ ${ }^{1}$ Chicago Medical School, Rosalind Franklin University of Medicine and Science, Chicago, USA \\ ${ }^{2}$ Rosalind Franklin University of Medicine and Science, Chicago, USA \\ Email: Patrick.Knott@rosalindfranklin.edu
}

Received 26 May 2015; accepted 19 July 2015; published 22 July 2015

Copyright (C) 2015 by authors and Scientific Research Publishing Inc.

This work is licensed under the Creative Commons Attribution International License (CC BY). http://creativecommons.org/licenses/by/4.0/

(c) (i) Open Access

\begin{abstract}
Barefoot running is increasing in popularity within the running community, yet the biomechanical differences compared to traditional shod running are not well understood. This study investigates the changes in spinal dynamics during the gait cycle of runners wearing traditional running shoes (shod) compared to those wearing no shoes (barefoot). Pedal force distribution, kyphotic angle, lordotic angle, and trunk inclination were measured during shod and barefoot gait at three different speeds on a treadmill. Subjects were examined using the DIERS Formetric 4D system and DIERS Pedoscan system. While running barefoot, pedal force distribution analysis showed that $\mathbf{2 1 . 0 \%}$ more force load goes through the forefoot vs. a $10.2 \%$ increase in forefoot force with shod $(p=0.0006)$. At $8.0 \mathrm{~km} \cdot \mathrm{h}^{-1}$ the average kyphotic angle was 1.6 degrees greater under barefoot conditions vs. shod conditions $(p=0.008)$. At $8.0 \mathrm{~km} \cdot \mathrm{h}^{-1}$ the average lordotic angle was $0.8 \mathrm{de}-$ grees greater under barefoot conditions vs. shod conditions $(p=0.05)$. Trunk inclination was 0.6 degrees and 0.8 degrees greater under barefoot conditions compared to shod conditions at natural speeds $(p=0.005)$ and $8.0 \mathrm{~km} \cdot \mathrm{h}^{-1}(p=0.015)$, respectively. Barefoot runners show an increased force load in the forefoot, eliciting a forefoot strike pattern while running barefoot compared to shod running, and it was also found that barefoot runners have reduced trunk inclination. These dynamic changes allow for a more spring-like effect for barefoot runners creating a less transient and slower rise in force. In contrast, a heel strike pattern yields a rapid and high impact collision between heel and ground. As a result, barefoot running translates into less stress on the joints of the lower extremity and back and therefore less risk of injury.
\end{abstract}

\section{Keywords}

Running Related Injury, DIERS, Barefoot Running, Scoliosis

\footnotetext{
*These authors contributed equally to this work.

How to cite this paper: Draus, C., Moravec, D., Kopiec, A. and Knott, P. (2015) Comparison of Barefoot vs. Shod Gait on Spinal Dynamics Using DIERS Formetric 4D and DIERS Pedoscan Systems. Open Journal of Therapy and Rehabilitation, 3, 70-76. http://dx.doi.org/10.4236/ojtr.2015.33010
} 


\section{Introduction}

Barefoot running is not a new phenomenon; in 1960, Ethiopia's AbebeBikila won the first of two consecutive Olympic gold medals and set a new world record all while running barefoot [1]. Recently, barefoot running has been increasing in popularity among runners, many of whom claim that running barefoot or using minimalist shoes will decrease injuries and improve performance. Researchers have begun to investigate these claims of increased performance and safety by analyzing the differences in pedal force distribution between full shod and barefoot runners. Specifically, their examinations have looked to see if shod running causes a heel strike pattern and if barefoot running elicits a forefoot strike pattern. In 2010, Lieberman et al. found that full shod runners tend to strike the ground heel first, while barefoot runners will to strike the ground forefoot first [2]. The difference in strike patterns is significant because when a runner's heel strikes the ground first, the foot absorbs a transient and instantaneous large force, which is subsequently transferred up the kinetic chain extending from the runner's lower extremity to the spine. In contrast, with forefoot striking, the impact is distributed over a greater area and therefore one body part does not absorb the whole impact of the runner's body weight. Consequently, the runner's lower extremity and spine receive less of a shock during impact [3].

Spinal health is often overlooked as an area of investigation when considering running injuries. However, runners can develop numerous back injuries including sciatica, lumbosacral strain, and potentially spinal stenosis over time [4]. If barefoot running leads to an increased forefoot strike pattern and this pattern decreases the force of impact running exerts on the kinetic chain, it would be interesting to consider the potential clinical significance that barefoot running can have on the spinal health of runners.

This paper aims to investigate this proposed clinical significance. Differences in pedal force distribution between shod and barefoot running were analyzed, specifically to observe the type of foot strike pattern (forefoot dominant or heel dominant) when the runner impacts the ground. Additionally, we examined potential differences in thoracic kyphosis, lumbar lordosis, and the trunk inclination angle between barefoot and shod running.

\section{Materials and Methods}

\subsection{Materials}

Dynamic posture data was obtained using the DIERS Formetric 4D analysis system (DIERS International $\mathrm{GmbH}$, Schlangenbad, Germany) in conjunction with a treadmill. Pedal force patterns were obtained using the DIERS Pedoscan system (DIERS International GmbH, Schlangenbad, Germany). This instrument was originally developed to monitor the spinal curvature of patients with scoliosis. When used to evaluate static posture, the device has been shown to provide reproducible measurements of the spine with standard deviations consistent with those obtained by standard radiographs [5]. The DIERS Formetric 4D system captures 50 picture frames per second for 5 seconds and converts these images into a reconstructed 3D map of the spine. The spinal dynamics are measured in each frame creating a real time representation of spinal movement as it occurs during the gait cycle.

\subsection{Inclusion and Exclusion Criteria}

Participants were recruited from Rosalind Franklin University of Medicine and Science and the surrounding area. Individuals over the age of 18 years old that read, understood and signed the IRB approval form were eligible to participate in this study. Inclusion was not based on sex, race, religion, insurance, or socioeconomic status. Participants were excluded if they had neurological or orthopedic conditions that would affect their gait. These pre-existing orthopedic conditions included joint arthroplasty, symptomatic ligamentous injury, spinal deformities, or other gait disturbances due to physical and/or neurological conditions.

\subsection{Participants}

Ten healthy participants were included in this study with males and females represented equally. Participants ranged in age from 25 - 38 years old, 1.57 - 1.85 meters tall and 52.2 - $95.3 \mathrm{~kg}$. Additionally, participants possessed a wide range of experience, from recreational runners to marathon and ultramarathon competitors. No participants had previous experience with barefoot running. 


\subsection{Experimental Setup}

Prior to collecting dynamic posture data, equipment was calibrated to participants on an individual basis. Participants were required to expose their backsides from the hairline to several centimeters inferior to the superior aspect of the gluteal cleft. Reflective markers were utilized to facilitate recognition of specific anatomic landmarks by the DIERS Formetric 4D system. The markers were manually placed on the C7 spinous process and bilateral posterior superior iliac spines of each participant. The accuracy of marker placement was verified by obtaining static posture data using the DIERS Formetric 4D system. This data provides a surface topographical map of participants' bodies and automatically locates the C7 spinous process and posterior superior iliac spines on the basis of relative elevation and depression, respectively. If the manual placement of markers was in disagreement with the computed location, markers were moved and a repeat static scan was performed to ensure agreement and proper placement.

\subsection{Experimental Procedure}

Participants were evaluated for pedal force distribution and dynamic posture including kyphotic angle VP-ITL (angle measured between the C7 spinous process and the thoracic-lumbar inflection point), lordotic angle ITLDM (angle measured between the thoracic-lumbar inflection point and the midpoint between lumbar dimples) and trunk inclination VP-DM (angle measured between the midpoint between the lumbar dimples and the C7 spinous process) (Figure 1). Participants were first instructed to walk on a treadmill at $2.9 \mathrm{~km} \cdot \mathrm{h}^{-1}$ while dynamic

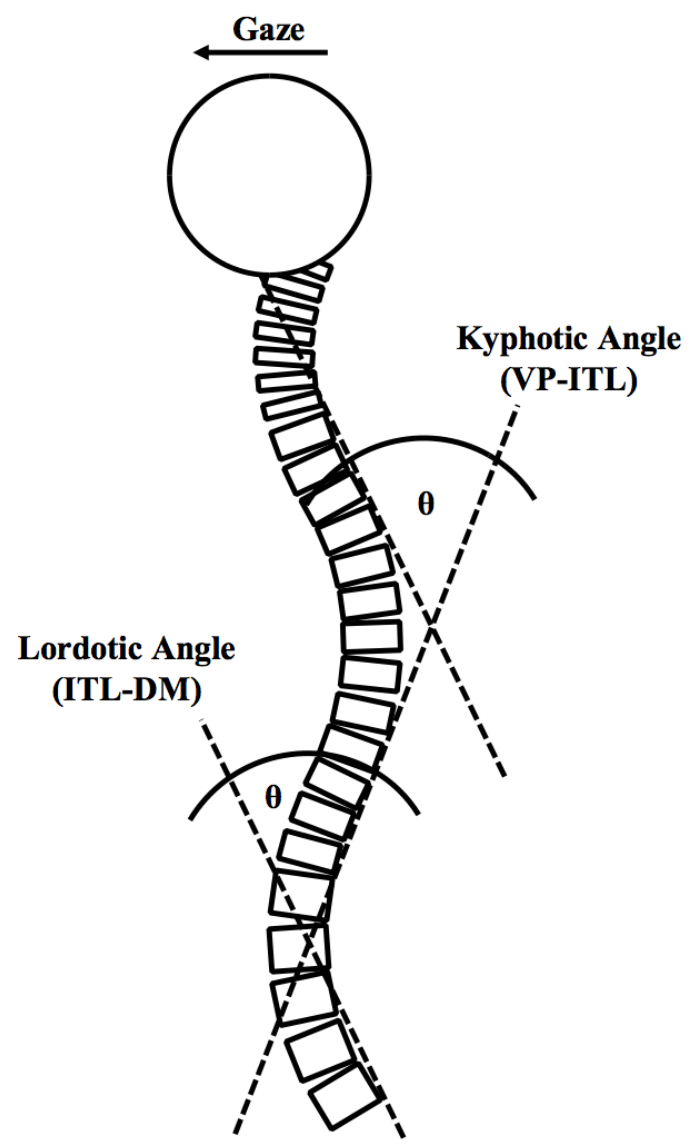

Figure 1. Schematic representation of several parameters of interest. Kyphotic angle (VP-ITL) is the angle measured between the C7 spinous process and the thoracic-lumbar inflection point. Lordotic angle (ITL-DM) is the angle measured between the thoracic-lumbar inflection point and the midpoint between lumbar dimples. The sagittal vertical axis, or trunk inclination (VP-DM) is the angle measured between the midpoint between the lumbar dimples and the C7 spinous process. A positive VP-DM indicates the subject is leaning forward over their spine, while a negative VP-DM indicates the subject is leaning backward. 
posture data was collected with the DIERS Formetric 4D system. Participants were then instructed to walk at a speed that they found natural across the DIERS Pedoscan system in order to obtain pedal force distributions. Next, participants were instructed to walk at the same speed that they found natural on the treadmill while dynamic posture data was collected. Participants were then asked run at a speed that they found natural across the DIERS Pedoscan system in order to obtain pedal force distributions. Finally, participants were instructed to run on the treadmill at $8.0 \mathrm{~km} \cdot \mathrm{h}^{-1}$ while dynamic posture data was collected.

Each participant was required to complete the above procedure both shod and barefoot. All dynamic measurements using the DIERS Formetric 4D system were recorded for 5 seconds and repeated until 3 successful trials were obtained. All pedal force distribution measurements using the DIERS Pedoscan system were repeated until 3 successful trials were obtained with each foot.

\subsection{Data Analysis}

The DIERS Formetric 4D and DIERS Pedoscan Systems were used to collect data from the subjects [6]. Regarding dynamic posture data, all trials were examined frame-by-frame to identify and remove outliers from the analysis. Outliers were defined as any point at which the DIERS Formetric 4D system was unable to accurately locate the C7 spinous process and/or posterior superior iliac spines bilaterally. Values for kyphotic angle, lordotic angle and sagittal vertical axis metrics were extracted from the remaining frames and averaged. A similar process was applied to the analysis of pedal force distributions in which case outliers were defined as a trial in which any portion of the foot strike was not detected by the DIERS Pedoscan system. Statistical significance was determined by one-way, paired Student's T-test.

\section{Results}

\subsection{Pedal Force Distribution}

When walking at speeds found to be natural, barefoot and shod gait yielded nearly identical pedal force distributions (Figure 2(a)). On average, 1.4\% and 1.6\% more load goes through the forefoot under barefoot and shod conditions, respectively $(\mathrm{p}=0.47)$. Alternatively, when running at speeds found to be natural, barefoot and shod gait yielded distinct pedal force distributions (Figure 2(b)). On average, 21.0\% and 10.2\% more load goes through the forefoot under barefoot and shod conditions, respectively $(p=0.0006)$.

\subsection{Dynamic Posture}

No statistically significant differences were observed in the kyphotic angle (VP-ITL) of subjects walking at 2.9 $\mathrm{km} \cdot \mathrm{h}^{-1}(\mathrm{p}=0.5)$ and at speeds found to be natural $(\mathrm{p}=0.42)$ (Figure 3(a)). However, at $8.0 \mathrm{~km} \cdot \mathrm{h}^{-1}$ it was found

Pedal Force Distribution

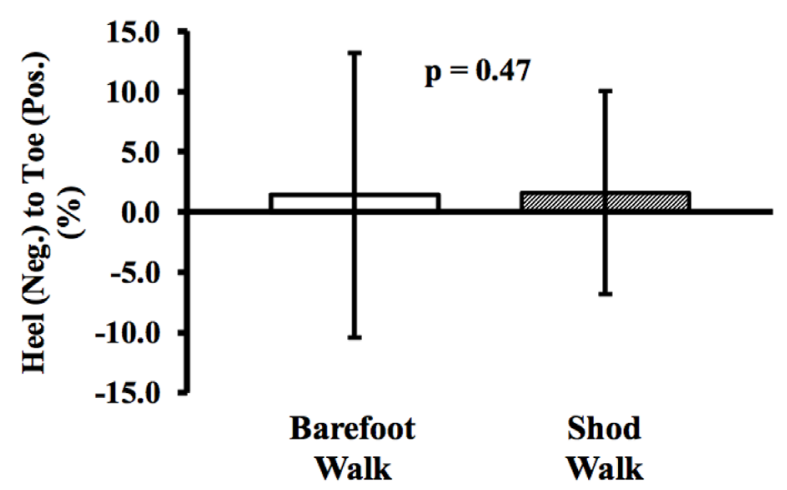

(a)
Pedal Force Distribution

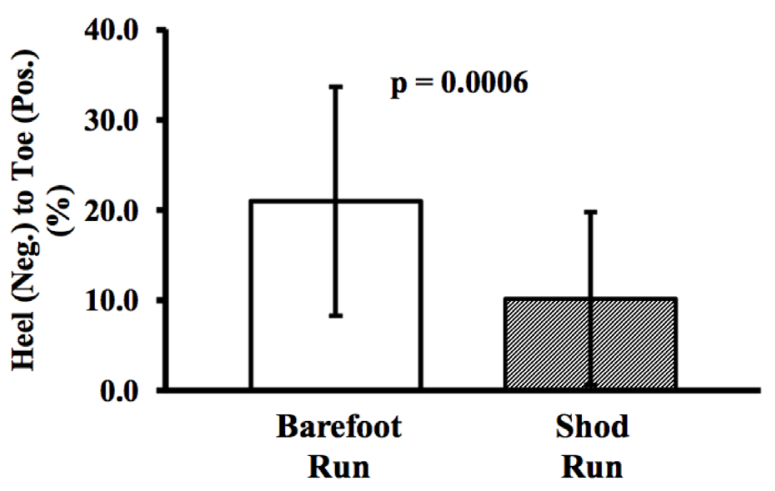

(b)

Figure 2. Comparison of barefoot vs. shod pedal force distribution $(\mathrm{n}=10)$. Negative values indicate a hindfoot-dominant pedal force distribution. Positive values indicate a forefoot-dominant pedal force distribution. Statistical significance was determined by one-way, paired Student's T-test. 


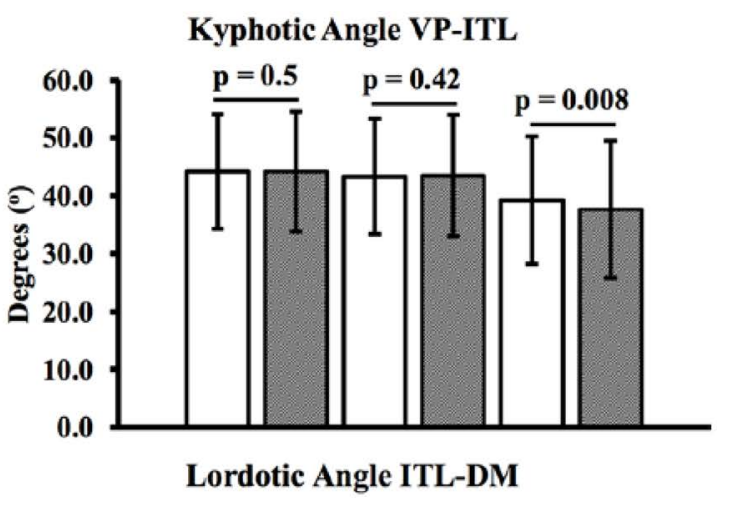

(a)

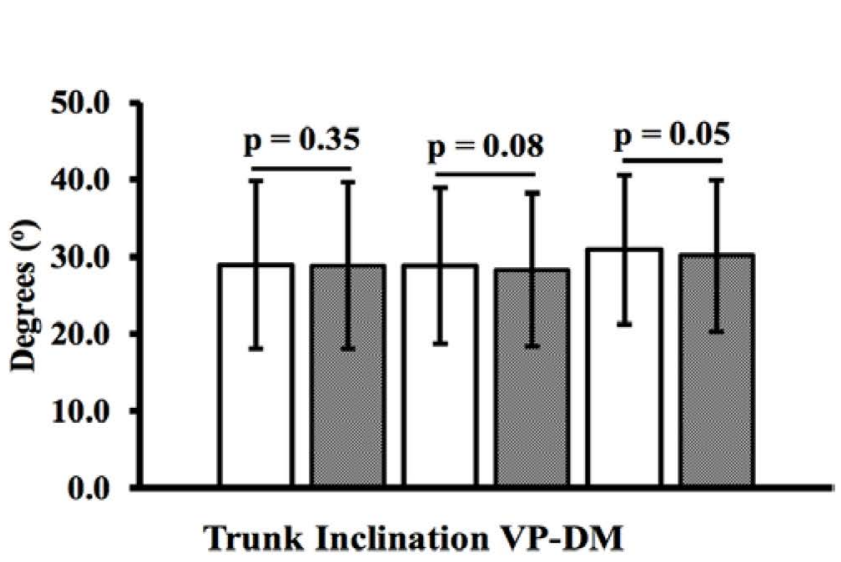

(b)

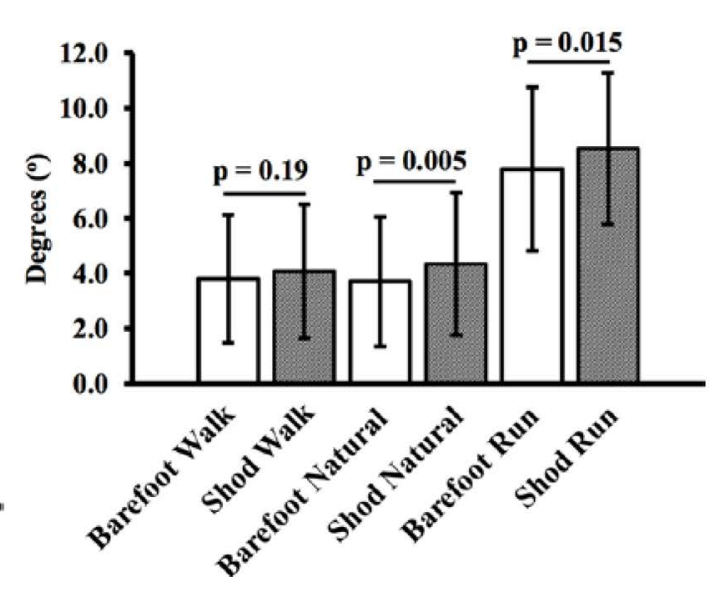

(c)

Figure 3. Comparison of barefoot vs. shod kyphotic angle, lordotic angle and sagittal vertical axis $(\mathrm{n}=10)$. Statistical significance was determined by one-way, paired Student's T-test.

that the average kyphotic angle was 1.6 degrees greater under barefoot conditions as compared to shod ( $\mathrm{p}=$ 0.008). Again, no statistically significant differences were observed in the lordotic angle (ITL-DM) of subjects walking at $2.9 \mathrm{~km} \cdot \mathrm{h}^{-1}(\mathrm{p}=0.35)$ and at speeds found to be natural $(\mathrm{p}=0.08)$ (Figure $\left.3(\mathrm{~b})\right)$. A statistically significant difference became apparent at $8.0 \mathrm{~km} \cdot \mathrm{h}^{-1}$ when the average lordotic angle was 0.8 degrees greater under barefoot conditions as compared to shod $(\mathrm{p}=0.05)$. Trunk inclination (VP-DM) was found to be similar at 2.9 $\mathrm{km} \cdot \mathrm{h}^{-1}$, however, at speeds found to be natural and $8.0 \mathrm{~km} \cdot \mathrm{h}^{-1}$, statistically significant differences were observed (Figure 3(c)). Trunk inclination was found to be 0.6 degrees and 0.8 degrees greater under barefoot conditions compared to shod at speeds found to be natural $(\mathrm{p}=0.005)$ and $8.0 \mathrm{~km} \cdot \mathrm{h}^{-1}(\mathrm{p}=0.015)$, respectively.

\section{Discussion}

The most important finding in this study was barefoot running predominantly elicited a forefoot striking pattern, while full shod running demonstrated primarily heel strike patterns. These findings are consistent with results from other researchers in the field. Lieberman et al. studied pedal force distribution on several different groups of runners: those that grew up as shod runners, those that grew up as barefoot runners and recently switched to shod running, those that had recently switched from shod to barefoot running, and those who have only run barefoot. The data showed that runners who had grown up as shod runners displayed greater rear foot strike patterns overall, but did generate flatter foot placements when running barefoot on hard surfaces. In contrast, those who grew up running barefoot or switched to barefoot running showed a predominant fore foot strike pattern [2]. The results of our study are in concordance with the findings of Lieberman et al.

The data also showed there was a disparity in forefoot strike vs. heel strike in participants during running vs. walking. When walking, participants showed $1.4 \%$ and $1.6 \%$ more load goes through the forefoot under barefoot and shod conditions, respectively. However when running, $21.0 \%$ and $10.2 \%$ more load goes through the fore- 
foot under barefoot and shod conditions, respectively. This discrepancy is primarily due to the dynamic differences of a walking gait compared to a running gait. When walking, the lower body is stiffer with locked knees, facilitating a greater propensity for heel strike. When running the gait is more like a spring, legs are bent, knees are unlocked, and the body is generating more force. The feet function more as tension stabilizers to absorb the impact and then spring the body forward, a movement that is better facilitated by a forefoot strike pattern [7].

The increased lordotic and kyphotic angles observed with barefoot running as well as the decrease in trunk inclination with barefoot running are statistically significant, but unlikely to be clinically relevant as the actual change in the angles is quite small. Data in the field on these specific parameters is sparse, but in 2013, Delgado et al. showed results which conflicted with our findings [8]. Delgado et al. examined the change in lumbar range of motion when runners change from rear foot strike to forefoot strike, specifically focusing on changes in the lordotic angle. The data showed that changing from rear foot strike to forefoot strike decreased overall range of motion in the lumbar spine. Additionally, the data showed that shock attenuation was greater in rear foot strike compared to a forefoot strike, thereby conflicting with the results of this current investigation and those of Lieberman et al. 2010 [8].

Studies have shown that barefoot runners typically exhibit a forefoot-midfoot strike pattern with initial contact with the ground, while runners in full shod make their initial contact with their heel [2]. Clinically, a forefoot strike pattern creates more of a spring-like motion, the impact is less transient and creates a slow rise in force that will dissipate throughout the runner's foot, allowing for a less severe reverberation up the rest of the body (knee, hip, pelvis, and spine). In contrast a heel strike pattern yields a rapid and high impact collision between heel and ground. The force can actually be up to 3 times a runner's body weight. This force is transferred up the runner's lower extremity and spine with every stride essentially creating a high impact trauma [3]. With full shod running, the heel strikes first and elicits an overall imbalance of weight distribution, causing less of the force of impact to be dissipated and absorbed throughout the entire body. This imbalance of force subsequently places an amplified strain on the body's natural pressure loading points such as the lower back, hip, and knees, potentially leading to injury at these places while running with a standard full shod shoe.

While our main finding was found in the analysis of pedal force distribution, the evaluation of kyphosis, lordosis and inclination angle also yielded statistically significant differences between barefoot and shod. Specifically for kyphosis and lordosis there were statistically significant differences seen when participants ran at 8.0 $\mathrm{km} \cdot \mathrm{h}^{-1}$ and for inclination angle differences were observed when participants moved at their natural walking speed. However, because the actual changes in the angles were so small, there is unlikely any major clinical significance to these findings, but they are still worthy of discussion. At $8.0 \mathrm{~km} \cdot \mathrm{h}^{-1}$ participants running barefoot had larger thoracic kyphotic angles (39.2 degrees barefoot vs. 37.6 degrees shod) and larger lumbar lordotic angles (30.9 degrees barefoot vs. 30.1 degrees shod). The larger thoracic kyphotic and lumbar lordotic angles suggest that while running barefoot there is a greater range of motion throughout the spine as compared to running full shod. This finding would be in contrast to Delgado's finding that lordotic range of motion was actually decreased with forefoot strike [8]. In this current investigation we cannot be sure if the participants were actually engaging in forefoot strike pattern as we were not able to measure pedal force distribution simultaneously with thoracic and kyphotic angle changes. One could infer however, that a greater range of motion within the spine of barefoot runners allows for an increased spring-like effect during their gait. More in depth research is required to follow up on this possibility.

Similarly, while the comparison of angles of inclination may not be great enough to have profound clinical significance (7.8 degrees barefoot vs. 8.5 degrees shod running), the overall observation that barefoot runners have smaller inclination angles can allude to changes in spinal dynamics. The angle of inclination is a measure of how far forward or backward one is leaning while they move and is closely related to how a runner lands on their foot. The smaller angle of inclination observed with barefoot running supports the clinical relevance of the statistically significant difference found in pedal force distribution. Our data has shown that barefoot runners, using their forefoot strike pattern have a smaller degree of inclination, meaning the barefoot runner is more upright during their stride. Landing upright and less forward will allow for a more balanced distribution of force throughout the body, minimizing anatomic stress points that may lead to injury, such as lower back pain.

Although statistically significant differences in spinal dynamics between barefoot and shod running were found, there were several limitations to our research. The sample size of this study was relatively small $(n=10)$ and could be increased to include more participants in an attempt to confirm the precision of our data. Furthermore, the DIERS Pedoscan pressure plate was separate from the treadmill that participants ran on during the 
collection of spinal dynamic data, and because of this the participants' running speed may have been slightly different between the two measurements. In addition, it is possible that because we could not measure spinal dynamics and foot pressure simultaneously, our participants may not have had the same pedal striking patterns on the treadmill as they did on the DIERS Pedoscan. To validate that the changes in pedal force distribution match the changes in spinal dynamics, it would be best to have a treadmill with a built-in DIERS Pedoscan system on the belt. Camera speed was another limiting factor for this research (frames/second). In this study, the camera speed only allowed for clear images to be captured at a maximum speed of $8.0 \mathrm{~km} \cdot \mathrm{h}^{-1}$. While it is possible that $8.0 \mathrm{~km} \cdot \mathrm{h}^{-1}$ is a natural speed for some runners, others participants in our study stated the maximum speed of $8.0 \mathrm{~km} \cdot \mathrm{h}^{-1}$ was not fast enough to allow for their natural running gait. A faster shutter speed of the camera will allow for greater running speeds therefore allowing participants to exhibit a more natural running style.

\section{Conclusion}

The main finding of our study was that barefoot runners showed an increased force load in the forefoot, eliciting a forefoot strike pattern while running barefoot compared to shod running. The impact of this research is that it is possible that barefoot running creates a natural instinct of protection, thus forming a running cycle that is less stressful on the body. As there is conflicted evidence in the field as to the differences in spinal dynamics between barefoot and shod running, further investigation is warranted. Specifically, it is imperative that the pedal force distribution measured simultaneously with the spinal dynamics to ensure a forefoot strike pattern is associated with the increased thoracic kyphotic and lumber lordotic angles as well as the decreased trunk inclination angle.

\section{References}

[1] A Brief History of Barefoot Running (2011). http://www.runnersworld.com/barefoot-running-minimalism/a-brief-history-of-barefoot-running

[2] Lieberman, D.E., Venkadesan, M., Werbel, W.A., Daoud, A.I., D’Andrea, S., Davis, I.S., Mang'eni, R.O. and Pitsiladis, Y. (2010) Foot Strike Patters and Collision Forces in Habitually Barefoot versus Shod Runners. Nature, 463, 531535. http://dx.doi.org/10.1038/nature08723

[3] Biomechanics of Foot Strikes \& Applications to Running Barefoot or in Minimal Footwear (2010). http://www.barefootrunning.fas.harvard.edu/index.html

[4] American Orthopaedic Society for Sports Medicine (2008) Running and Jogging Injuries. http://www.sportsmed.org/uploadedfiles/content/patient/sports_tips/st\%20running\%20and\%20jogging\%2008.pdf

[5] Frerich, J.M., Hertzler, K., Knott, P. and Mardjetko, S. (2012) Comparison of Radiographic and Surface Topography Measurements in Adolescents with Idiopathic Scoliosis. The Open Orthopaedics Journal, 6, 261-265. http://dx.doi.org/10.2174/1874325001206010261

[6] DIERS International, GmbH (2014) DICAM Version 2.4.5. EU Patent, DIERS International GmbH, Schlangenbad, Germany.

[7] Maffetone, P. (2012) Walking vs. Running: Why These Gaits Are Not the Same. http://naturalrunningcenter.com/2012/06/21/walking-vs-running-gaits/

[8] Delgado, T.L., Kubera-Shelton, E., Robb, R.R., Hickman, R., Wallmann, H.W. and Dufek, J.S. (2013) Effects of Foot Strike on Low Back Posture, Shock Attenuation, and Comfort in Running. Medical Science Sports Exercise, 45, 490496. http://dx.doi.org/10.1249/MSS.0b013e3182781b2c 